\title{
An introduction to E-Prime
}

\author{
Laurence Richard \\ Miami University
}

\author{
Dominic Charbonneau \\ Université de Montréal
}

When running an experiment, precision is essential to ensure results are as exact as possible. Thus, computers, which offer endless accuracy, have become an inevitable tool to design experiments. To avoid programming from scratch for each new situation, a program, E-Prime, has been created to ease the conception of experiments. E-Prime, developed by PSTNet, offers a user-friendly interface that makes typical experiments easy to create. This paper shows how to effortlessly create an experiment with E-Prime, followed by a simple example.

E-Prime (2004) is a program designed to facilitate the conception of any experiment that uses a computer as an interface between the subject and the experimenter. It can also run the experiment, collect the results, do some basic data analysis and export said data. These options are parsed through the five programs that constitute E-Prime: E-Studio, E-Run, E-DataAid, E-Merge and E-Recovery.

\section{E-Studio}

E-Studio is the subprogram of E-Prime dedicated to the creation of experiments. It is the main program that comes with E-Prime.

\section{Interface}

E-Studio's easy to use interface is composed of four sections, as shown in Figure 1: Structure (1), Toolbox (2), Properties (3) and Worksheet (4). By dragging and dropping, it is possible to add any of the tools from the Toolbox to the Structure of the experiment. This also allows one to easily adapt an experiment that is already programmed by adding or deleting elements from the Structure or by selecting the elements to change in the Structure section and modifying them in the Worksheet.

\section{Structure}

The structure window gives an overview of the experiment being built, as shown in Figure 2.

The structure of the experiment should be thought through before even opening E-Prime. In an experiment, it is often easier to design starting with the more simple elements, such as the trial. Contrarily, it is easier, in EStudio, to start with the main concept and slowly work down to the trial. Some basic elements can be seen in the structure window such as the frames, the trials, the blocks and the complete experiment.

Frames:A frame is an event during the experiment. It can either be a slide with text or pictures shown to the subject or simply a delay. For the subject, an experiment is simply a sequence of frames. Each frame can have a fixed duration, be terminated by an external event or both. "Both" would be where a frame should be ended by an external event, such as the subject hitting the space bar, but with a limited time to do so.

Trials: Trials are where the real testing happens. They consist of an organized set of frames in which the stimuli are presented. Each trial will show different stimuli according to the goal of the experiment. In E-Prime, a template is programmed for the series of trials and the specifics of each trial are then listed.

Blocks : A block is a group of trials. Usually, an experiment will include a few blocks that will often be exactly the same. It is within the blocks that all trials necessary to collect all the data are listed. The blocks can also include an introductory frame and a conclusion one. These frames can be used as pauses during the experiment. They can also inform the subject of his or her progress by stating the number of blocks left.

\section{Complete experiment}

With all the blocks, the trials and the frames, the 


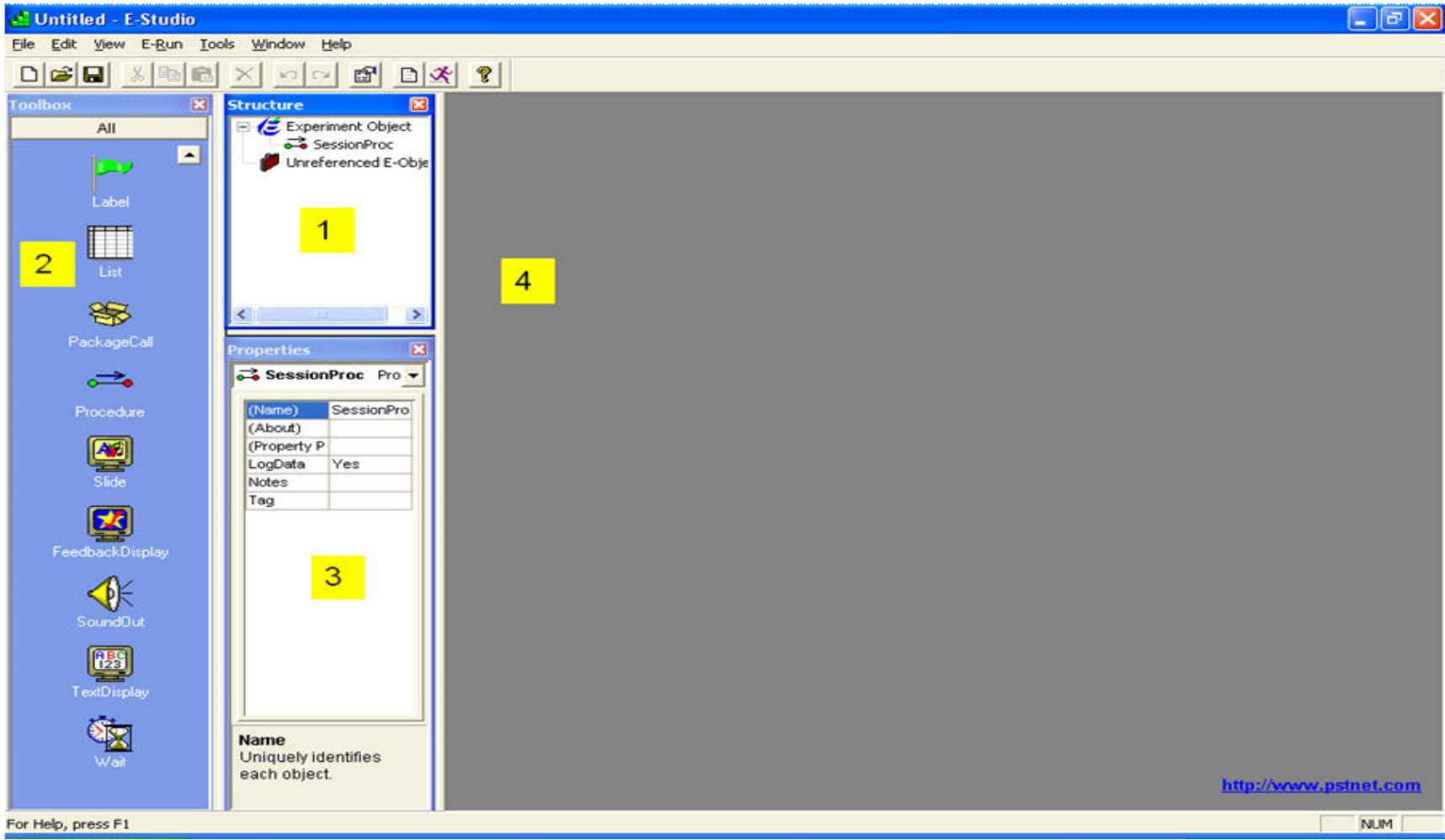

Figure 1: E-Studio's interface

experiment is complete. Only a few introductory frames with instructions and concluding remarks on other frames are missing to have the final product.

\section{Toolbox}

The toolbox contains all the objects that can be used in the experiment. In the structure window, the tools can be identified through the icons presented. The tools will be organized, as shown in the structure window, to create the experiment. Some of the tools can be grouped by their functions, while others are a class of their own.

Frames : There are quite a few tools that are used as frames.

Text display: The text display is a slide shown to the subject that can only contain text. It has many properties, as described in Table 1.

Image display: This frame presents images to the subject. It has practically the same properties as the text display.

Slide: The slide is far more polyvalent as it allows the user to manipulate text, images and sounds on the same frame. It is presented, much like the text display and the image display, to the subject at a specific time. The three small icons at the top of the frame, as shown in Figure 3, are used to insert any of the three elements. The slide's properties are close to the text display's properties.

Feedback display: The feedback display, as stated by its name, presents feedback to the subject. Feedback can be given about any action of the subject, as long as it is specified in the Input Object Name. Feedback can also present different information such as accuracy, response time, dispersion measures, etc. Accuracy can be defined by any of these three options: correct, incorrect or no response.

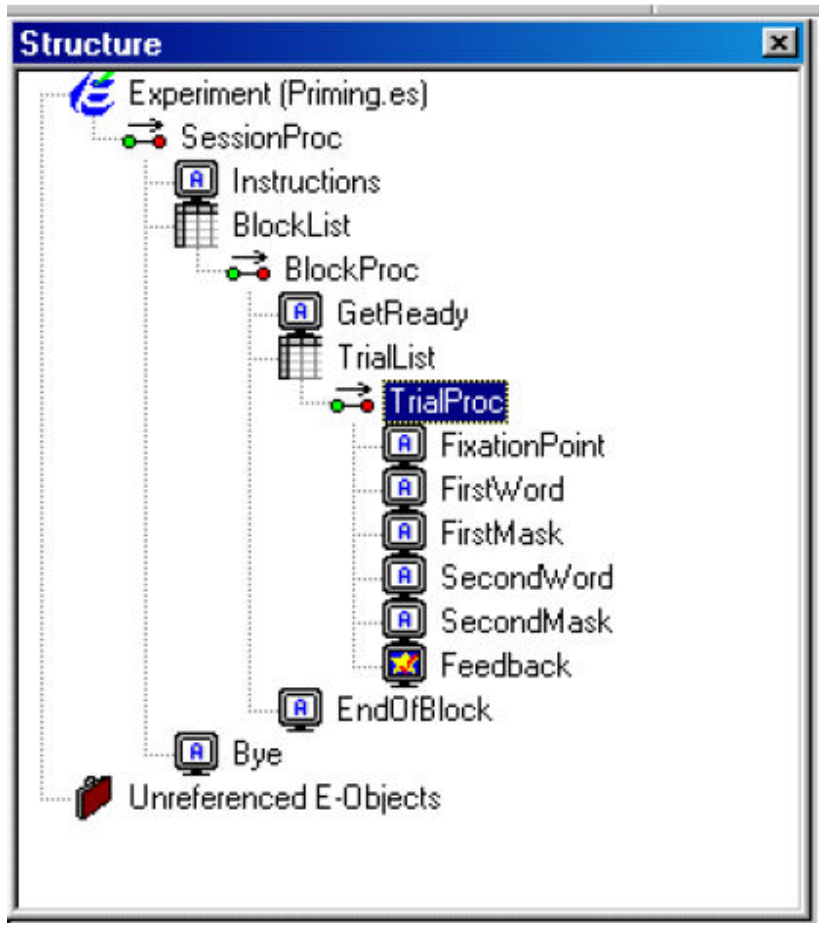

Figure 2: Example of the structure window 
Table 1: Text Display's Properties

\begin{tabular}{ll}
\hline General tab & $\begin{array}{l}\text { This shows the text which will be } \\
\text { displayed. }\end{array}$ \\
\hline $\begin{array}{l}\text { All the other options in this and the frame tab } \\
\text { modify the properties of the text. }\end{array}$ \\
\hline Duration/Input tab \\
\hline Duration & $\begin{array}{l}\text { Determines the length of the } \\
\text { presentation. }\end{array}$ \\
\hline Data logging & $\begin{array}{l}\text { To specify the kind of answer } \\
\text { expected from the subject. }\end{array}$ \\
\hline Devices & $\begin{array}{l}\text { To specify the device that will be } \\
\text { used to answer (ex: keyboard). }\end{array}$ \\
\hline Allowable & $\begin{array}{l}\text { To specify the keys used to } \\
\text { answer. All the other keys will be } \\
\text { disabled. }\end{array}$ \\
\hline Correct & $\begin{array}{l}\text { To specify the correct answer. } \\
\text { answer. }\end{array}$ \\
\hline Time limit & $\begin{array}{l}\text { To specify what will happen once } \\
\text { the frame ends. It can either stops } \\
\text { there (terminate), jump to a label } \\
\text { or do nothing (none). }\end{array}$ \\
\hline End action
\end{tabular}

Wait: This frame inserts a delay between two other objects. It does not have any visual or auditory displays; it only suspends the experiment for a specified length of time. Instead, the last frame will be displayed. The main property is Duration under the DurationInput tab, which dictates the length of the wait.

Procedure: One of the more important objects is the procedure, which organizes the frames and tools.

The objects from the toolbox can be dragged and dropped along the line drawn across the window. Afterwards, simply editing the different objects will create the experiment. It is also through the addition of a procedure that it is possible to change the level of specificity, as in going from the blocks to the trials. The procedure is used to create the complete experiment, then the blocks at a lower level and, finally, the trials.

The procedure only has one property: log data. It's used to prevent the recording of data during this specific procedure which might be useful for, among other examples, a practice set.

Since programming every trial would be extremely cumbersome, the procedure becomes a template. It is through the use of a list that the template will be modified with each trial and, in the end, will create the experiment.

List: The lists dictate how and how many times the procedures will be repeated. Mainly, they are used to create the blocks by housing the information specific to each trial. It is also with lists that the blocks are repeated. A list

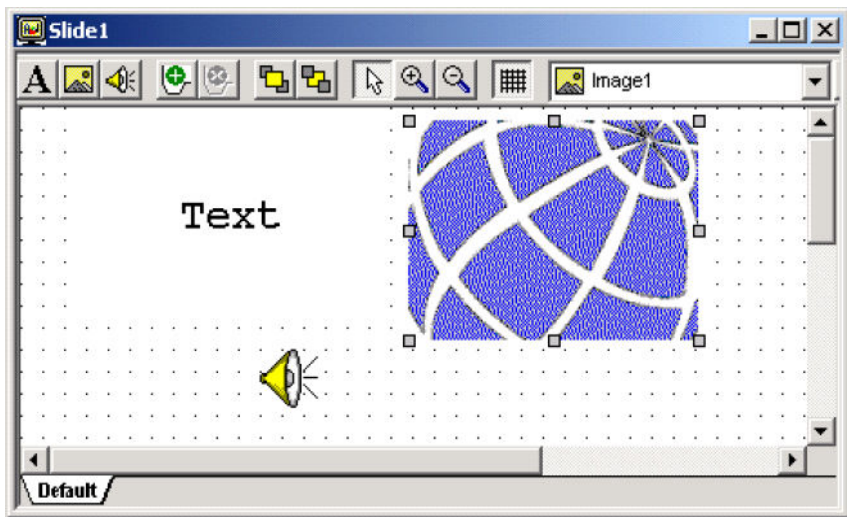

Figure 3: Slide window

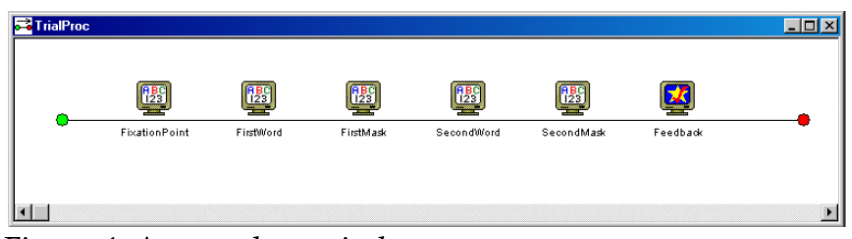

Figure 4: A procedure window

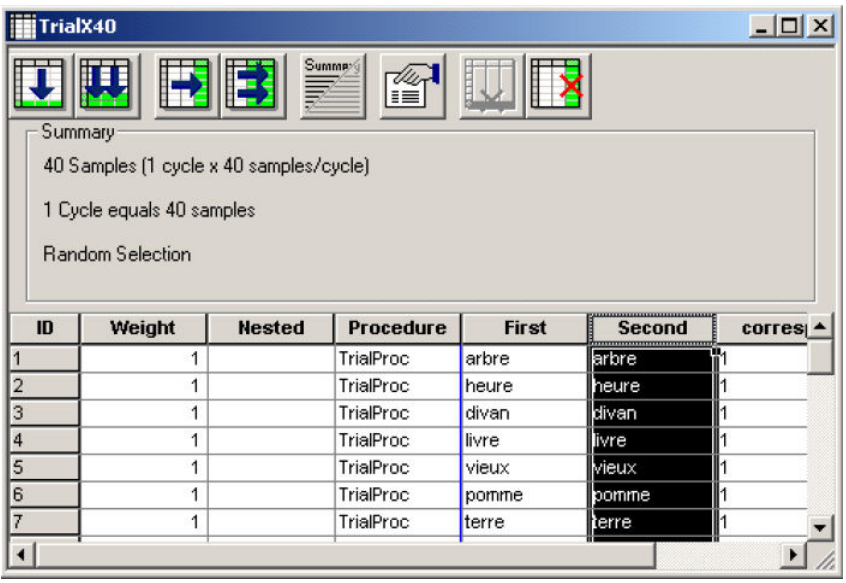

Figure 5: A list window

minimally contains three columns. One is to specify the procedure that should be displayed. Most of the time, all the rows will have the same procedure name, but if some trials are different from the rest and require a different procedure, this is where it should be noted. Next is a column for the weight an element should have (i.e. the number of time it is showed to the subject compared to the other elements). And, finally, a column called "Nested" to specify if there is another list under the present list. Other information can be added such as the stimuli used, the response expected, the order in which the elements should be presented, to name only a few. The list object has a few properties, as listed in Table 2.

In the list window, shown in Figure 5, there are a few simple tools. The table itself works somewhat like an excel worksheet.

The first two options respectively add one row or 
Table 2: List's Properties

\begin{tabular}{cl}
\hline \multicolumn{1}{l}{ Selection tab } & \\
\hline Order & $\begin{array}{l}\text { To specify the way each line will } \\
\text { be selected. }\end{array}$ \\
\hline $\begin{array}{l}\text { Reset } \\
\text { sampling }\end{array}$ & $\begin{array}{l}\text { To specify after how many lines } \\
\text { the list should be reset. }\end{array}$ \\
\hline Exit list & $\begin{array}{l}\text { To specify after how many trials } \\
\text { the list should be ended. }\end{array}$ \\
\hline
\end{tabular}

Table 3: Sound Out's Properties

\begin{tabular}{cll}
\hline General tab & Filename & $\begin{array}{l}\text { To insert the name of the } \\
\text {.wav file that will be used. }\end{array}$ \\
\cline { 2 - 3 } & Max length & $\begin{array}{l}\text { To specify the maximum } \\
\text { length of the sound. }\end{array}$ \\
\hline Duration/Input tab & \\
\hline Duration & $\begin{array}{l}\text { To specify the duration of } \\
\text { the sound. }\end{array}$ \\
\hline
\end{tabular}

Table 4: Examples of trials

\begin{tabular}{llll}
\hline Primer & Probe & Living & Congruency \\
\hline Nurse & Doctor & 1 & 1 \\
Knife & Driver & 1 & 2 \\
Bread & Butter & 2 & 1 \\
Passenger & Hammer & 2 & 2 \\
\hline
\end{tabular}

multiple rows. The next two do the same thing, but for columns. The next one governs the summary section. The last two delete a row or a column.

Other tools : There are a few other tools that can be used in E-Studio.

In Line: If the experiment being created has particulars not included in E-Studio, the In Line tool can be useful. Indeed, In Line is a blank page in which one can program

Figure 6: Structure of the priming experiment

\begin{tabular}{|c|c|c|c|}
\hline \multicolumn{2}{|c|}{ The Priming Experiment } & Duration & Description \\
\hline \multicolumn{2}{|c|}{ Instructions } & Until spacebar & \\
\hline \multicolumn{4}{|c|}{10 Blocks composed of } \\
\hline & GetReady & Until spacebar & \\
\hline \multicolumn{4}{|c|}{40 trials of } \\
\hline & FixationPoint & $800 \mathrm{~ms}$ & a "+" centered \\
\hline & FirstWord & $100 \mathrm{~ms}$ & two lines above "+" \\
\hline & FirstMask & $34 \mathrm{~ms}$ & "\#\#\#\#\#" \\
\hline & SecondWord & $100 \mathrm{~ms}^{*}$ & two lines above "+" \\
\hline & SecondMask & $2000 \mathrm{~ms}$ & "\#\#\#\#\#" \\
\hline & Feedback & $1500 \mathrm{~ms}$ & \\
\hline \multirow{3}{*}{ Bye } & EndOfBlock & Until spacebar & \\
\hline & & $10 \mathrm{~s}$ & \\
\hline & ${ }^{*}$ But, keeps lo & $2000 \mathrm{~ms}$ & \\
\hline
\end{tabular}

using the E-Basic code. This sheet can be used anywhere in the experiment. The only property is log data. It allows one to indicate if there's any data that should be recorded from this tool.

Sound Out: This object implements a sound within the experiment and, accordingly, doesn't have any visual presentation. There are a few properties associated as listed in Table 3.

Label : The label tool is used to mark a point in the time line. It is only useful when one wants to jump to a certain point in the experiment. It doesn't have any other properties than its placement.

Package call This tool is used to connect an E-Prime experiment to another device such as an EEG or an EKG.

\section{Properties}

This window shows the properties of the selected object, as seen in Figure 1, number 3. While useful, it can sometimes cause mistakes. It is indeed important to make sure that the object being modified is really the object requiring a change.

\section{Worksheet}

The worksheet is the main window where you see what the subject will see. It is shown in Figure 1, where it is identified as number 4 . It displays the visual part of the selected object.

\section{Example of an experiment}

To clarify all the notions on E-Studio here is an example: the Priming Experiment. In this experiment, the participant has to identify the probe word as being living or non-living. Before seeing the probe word, a prime word, shown too quickly to consciously see it, is presented. The prime word is either congruent (both of the same category) with the probe word or incongruent (both of different categories). The basic structure of the experiment is shown in Figure 6. 


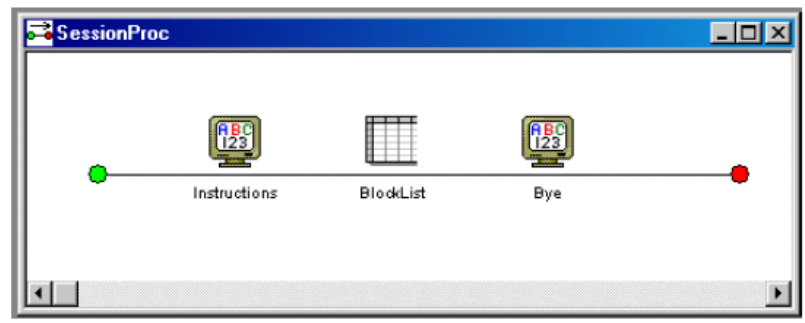

Figure 7: Session Procedure

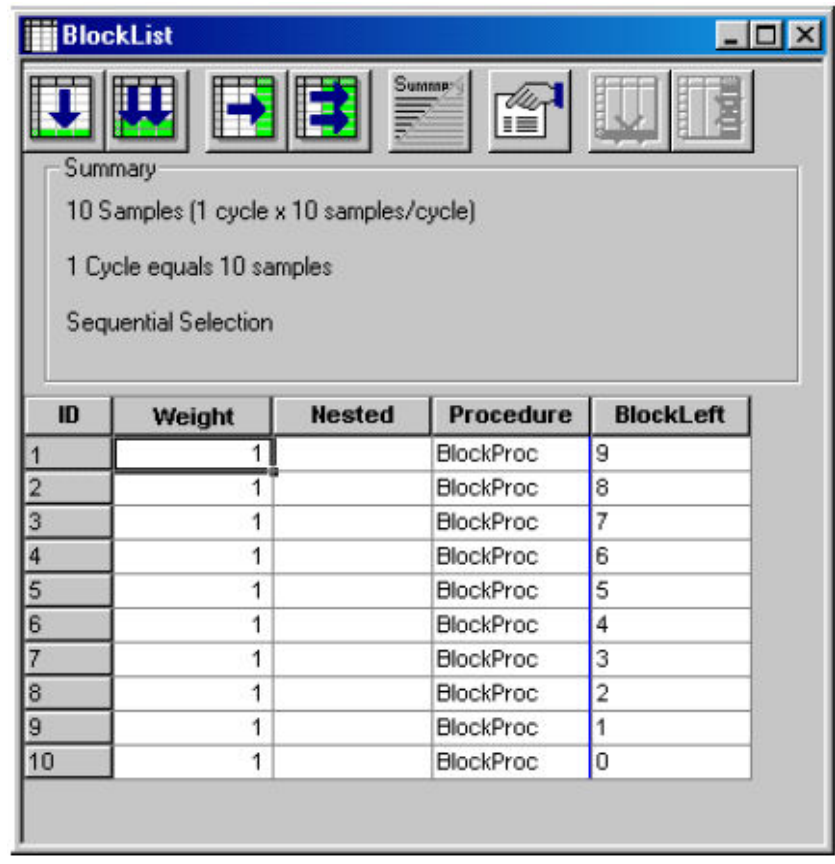

Figure 8: List interface

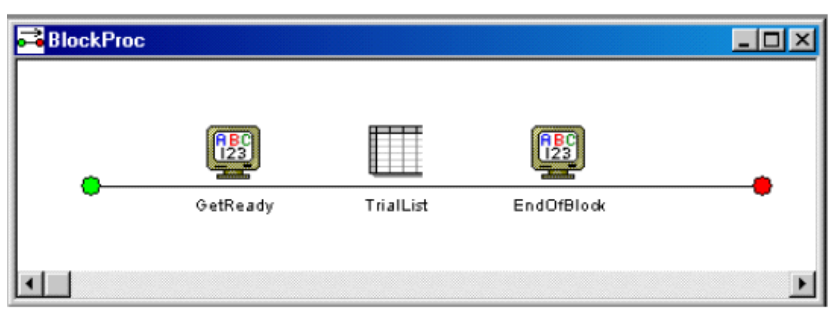

Figure 9: Block Procedure

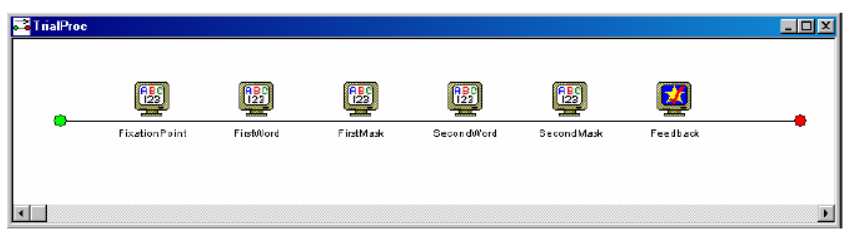

Figure 10: Trial Procedure

The trials will contain two stimuli each. Table 4 shows four examples of trials.

\section{Creating the structure}

Once the main structure of the experiment has been designed, the actual programming in E-Studio can start. First, to create the main frame, a procedure should be used. It can be created by double-clicking on the "Procedure" icon in the toolbar. Once the window is open (Figure 7) two text displays, one for the instruction and one for the end, are to be placed along the lines in the window by simply dragging and dropping them around a list. The text displays can be renamed for more clarity.

Next, a sublevel is created through the list. To achieve this, a new procedure is added in the list tool. By doubleclicking on the Blocklist object, its properties window appears. First, because the experiment consists of ten blocks and the number of lines should match, one can click on the second icon to add nine lines. Second, the "Procedure" column should present the name of the block procedure, here, in Figure 8, named BlockProc. The BlockLeft column of Figure 8 is used to indicate, to the subject, the number of blocks left.

By entering the name of a new procedure in the list, it is automatically created and the new level is added. Through the same process used for the first procedure, a template for each block is designed with two slides and a list (Figure 9).

In this new list, 39 lines are added since the design of the experiment demands 40 trials. The procedure column should show the trial procedure "TrialProc". In this new procedure, the template for each trial is found, as in Figure 10.

The structure of the experiment is now complete and clearly shown in the structure window (Figure 11).

\section{Changing the tools}

Each tool should have its properties changed to best fit the experiment. The "Instructions", "GetReady" and "EndOfBlock" frames should be ended by the participant hitting the spacebar. To achieve this, the Duration property, under the Duration/Input tab, should be set at "(infinite)" and the Allowable property at "\{SPACE\}" after the Keyboard has been selected in the Device(s) window (Figure 12).

For the two list objects, the selection order has to be specified. This can be done through the Selection tab in their properties window, in the Order command. The block list has to be set at Sequential while the trial list needs to be at Random.

In the trial procedure, the "FixationPoint", "FirstMask" and "SecondMask" frames need five lines for their specific displays. The center line is for a cross sign, which is the fixation point. The "FirstMask" will also show a "\#\#\#\#" sign on the first line while the "SecondMask" will show the same sign, but on the fifth line.

The two other slides, "FirstWord" and "SecondWord", show the actual stimuli. To achieve this, the "FirstWord" 


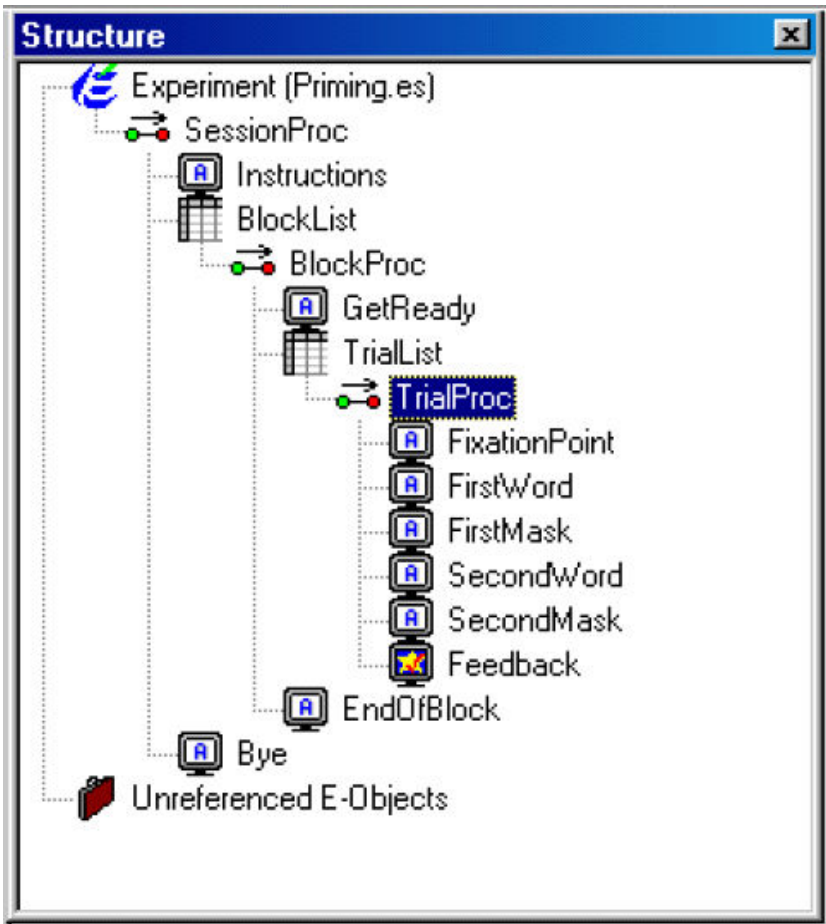

Figure 11: Structure Window

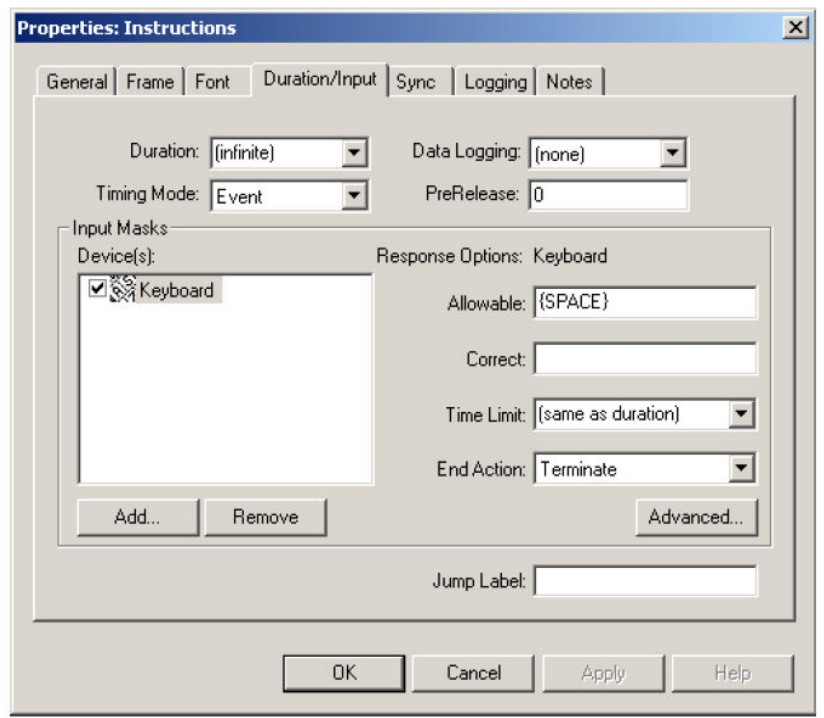

Figure 12: Duration/Input tab

slide will contain the five lines and the fixation point from earlier but, also, the word "[Primer]" situated on the first line. The same thing stands for the "SecondWord" slide, but the word is "[Probe]" and written on the fifth line. These two words will change with each trial to show the words included in the list. The list needs two more columns, named Primer and Probe to host the stimuli, as shown in Figure 13.

When the second word is presented, data has to be collected. First, the correct response has to be specified in the trial list under the name Living. The Congruency column of

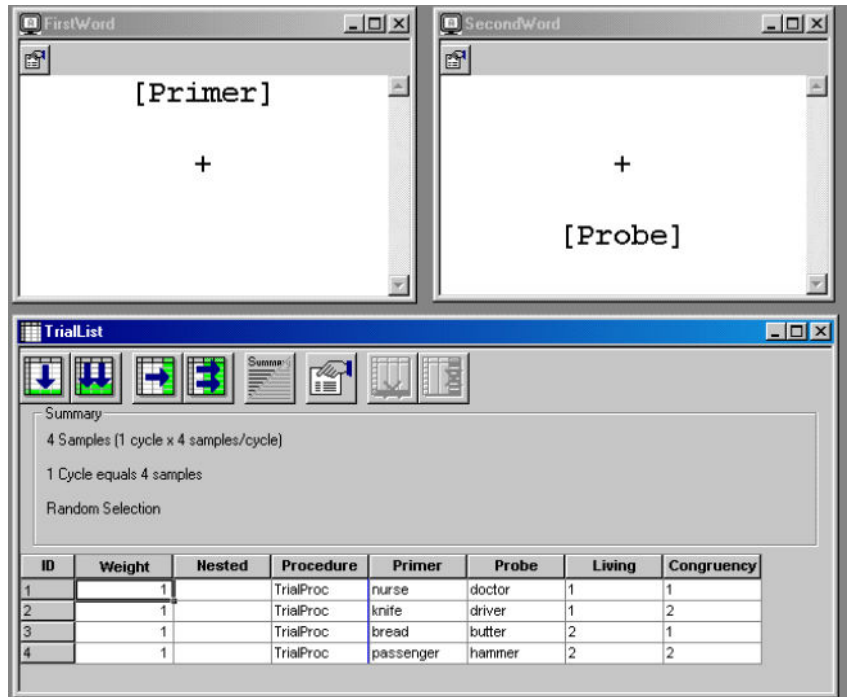

Figure 13: Trial list, "FirstWord" slide and "SecondWord" slide

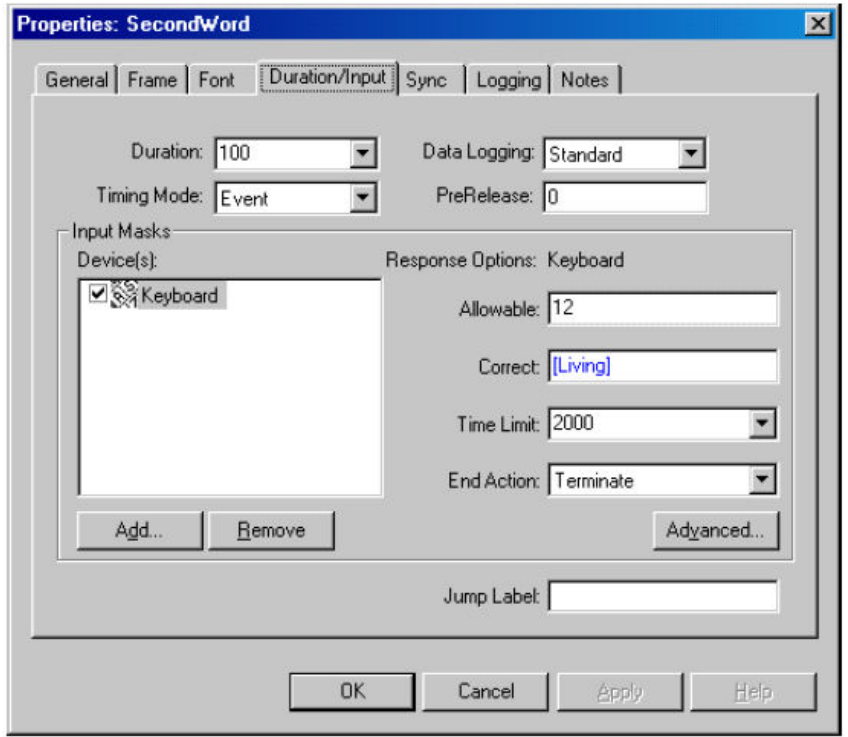

Figure 14: "SecondWord" slide's properties

Figure 13 was created only for analysis purposes and doesn't have any function in the experiment itself. In the "SecondWord" slide, in the properties window, under the Duration/Input tab, once the keyboard is selected as the device, the allowable keys are to be specified. In this case, both 1 and 2 are allowed and should be noted as 12 in the "Allowable" field. Also, the Correct field, just under the Allowable one, has to show [Living]. This refers to the columns containing the correct answers for each trial, as shown in Figure 14.

The time limit set at 2000 indicates that the participant has two full seconds to answer before the frame disappears.

Finally, the feedback display object has to be adjusted. The Input Object Name property has to show the "SecondWord" slide so that it can present the feedback for the right object. 


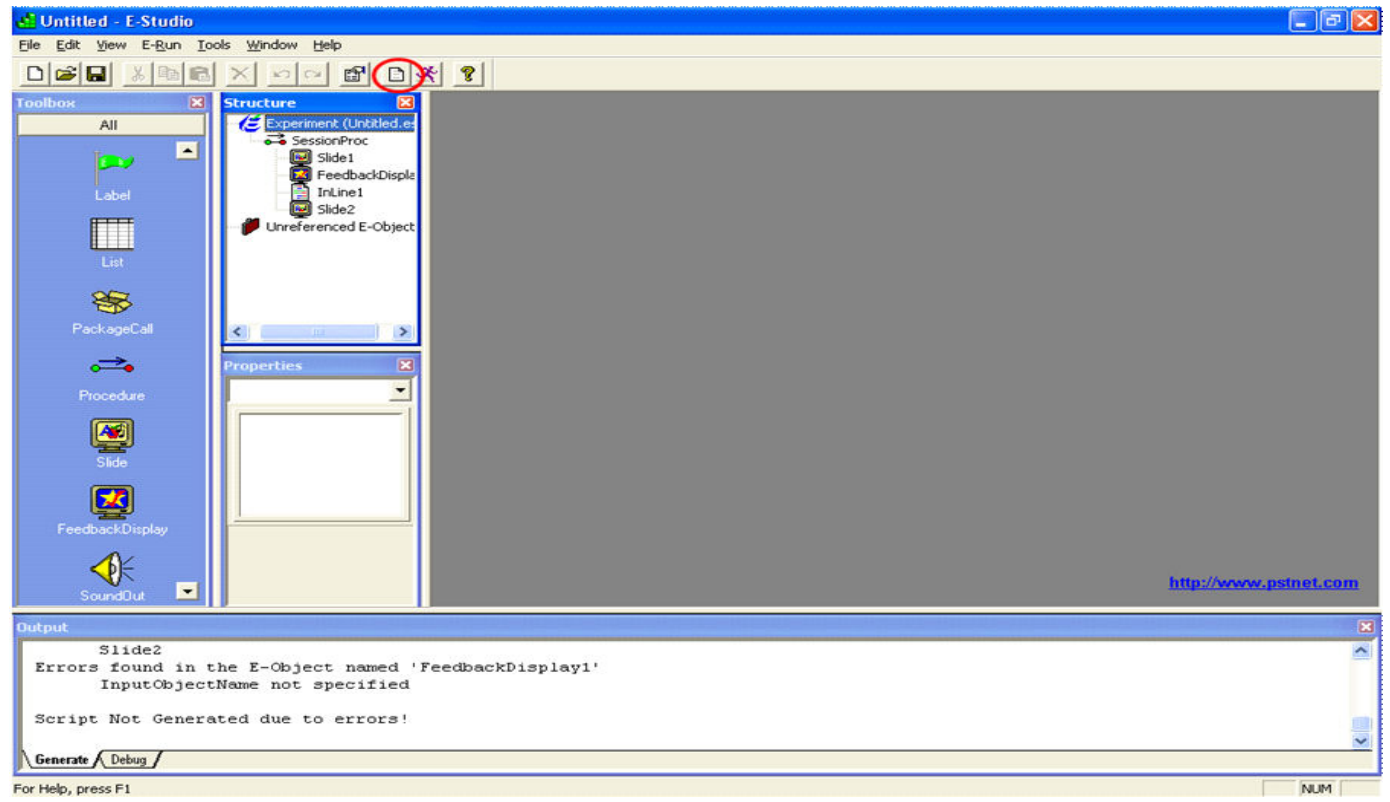

Figure 15: Testing the experiment

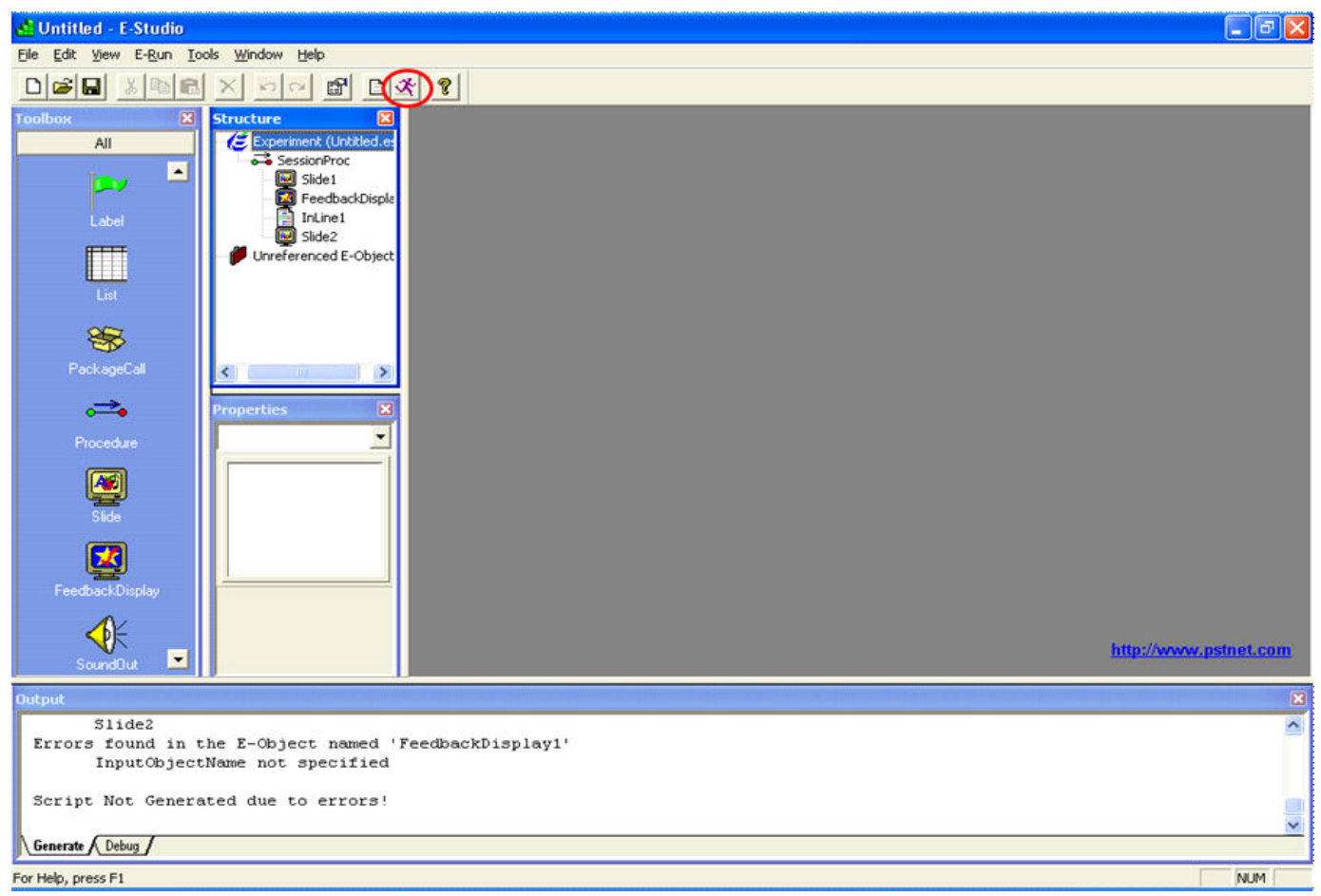

Figure 16: The Run button

\section{Testing the experiment}

The experiment is now complete and can be compiled. This option shows every mistake it finds. This process is activated through the Generate button, at the top of the screen, as shown in Figure 15. The errors found by E-Prime will be displayed in a new window named "Output" at the bottom of the screen, again shown in Figure 15. But, even though it is meant to find mistakes, it will not necessarily catch all of them and so the experiment should always be tried before beginning testing.

\section{E-Run}

The E-Run subprogram is designed to simply run the experiment. It is perfect for testing as it can be used without the licensed key. This way, the testing can be done on many 


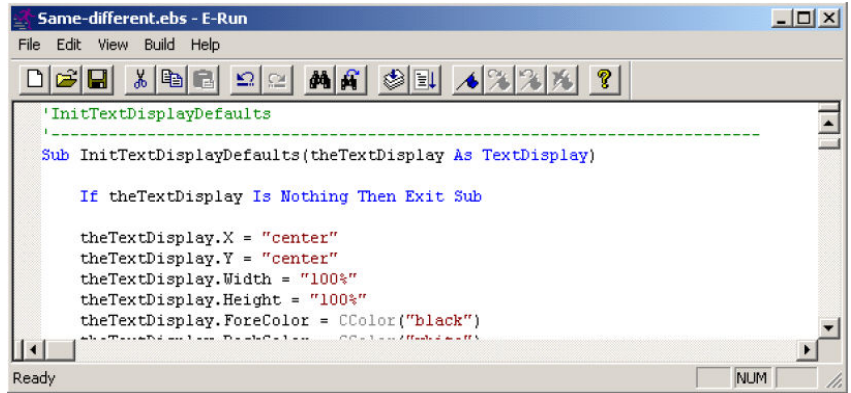

Figure 17: Opening window of E-Run

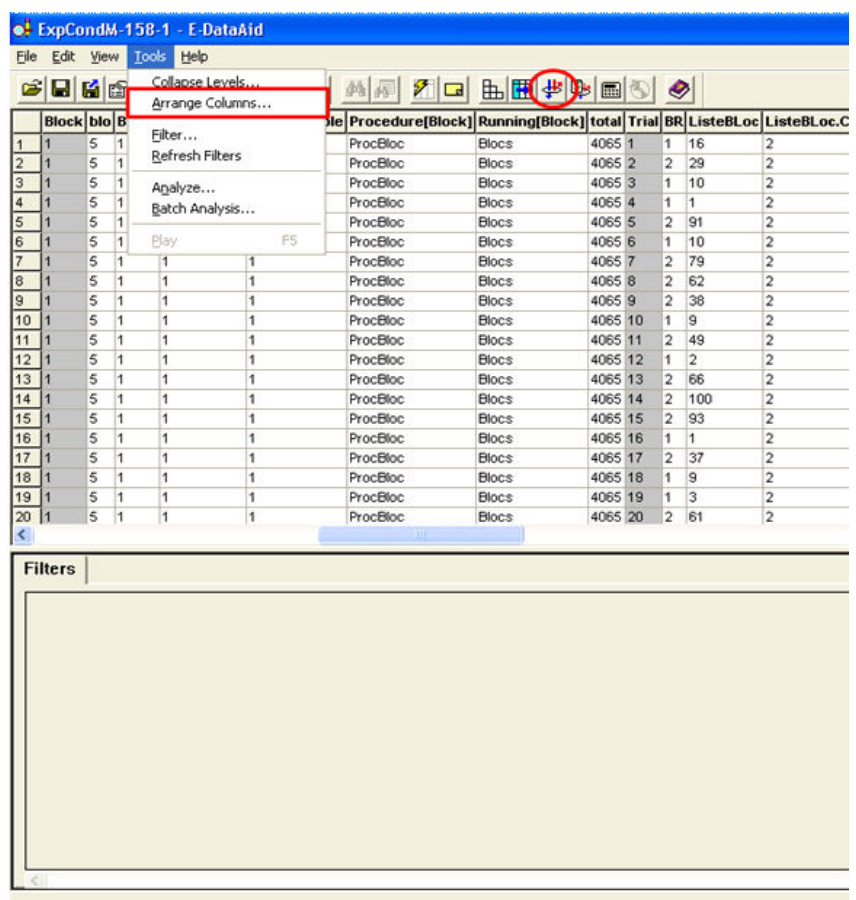

Figure 18: The E-DataAid interface

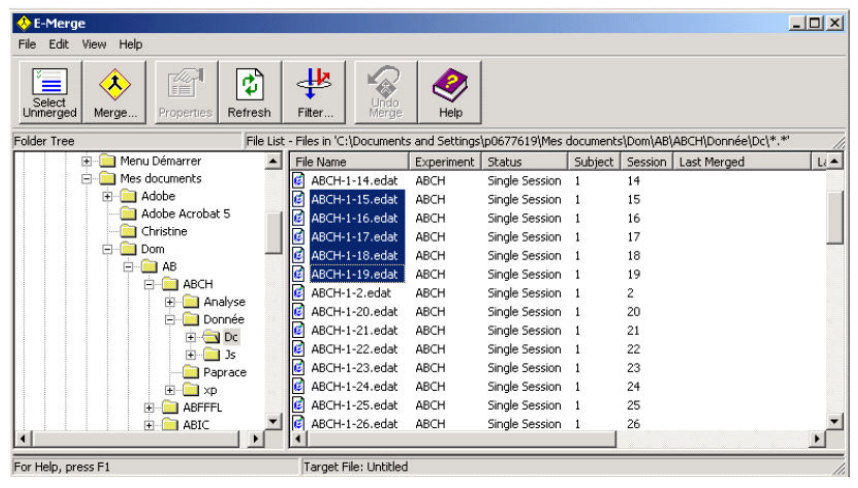

Figure 19: The E-Merge interface

computers simultaneously. The Run button starts the experiment. It is situated at the top of the screen, as shown in Figure 16. Once run through, it automatically creates two files: an .edat file and a .txt file. Both of these files contain the data gathered during the experiment. The edat file will be used by the E-DataAid subprogram described below. The .txt file is used by the E-Recovery subprogram, also described below. That is also the case if the experiment runs with E-Studio. Once the experiment has begun, it can only be aborted by pressing Ctrl+Alt+Shift.

Each experiment has an .ebs file, which is the E-Run file. This file shows the experiment in E-Basic code, as seen in Figure 17. While the experiment can be modified through this window, it is not the most practical way to do so.

\section{E-DataAid}

This program shows the data collected, as presented in Figure 18. It uses the .edat file created after running the experiment. It is a useful program that offers a few simple functions to modify the data, but nothing more. It is mainly practical to delete unnecessary information.

Each data file contains a lot of information under many columns. Every column is a different variable and many of those are useless. Unfortunately, it is not possible to stop EPrime from logging those variables, but they can easily be deleted without losing any information. With the Arrange columns option, in the Tools menu (see Figure 18), one can choose the variables one wants to keep. It is also possible to filter the lines with the Filter option, shown in Figure 18. For example, one could choose only one block for further analysis. All the columns taken away are actually only hidden and can be shown again with the same tools.

Since E-DataAid is somewhat limited, it is probably easier to export the information. Through the Export function in the file menu, the format compatible with other data analysis programs can be chosen.

\section{E-Merge}

When experimenting with many subjects, it is easier to do any analysis on the whole of the data accumulated. To create one file with information from all the subjects, EMerge can be used. Its interface is quite simple, as shown in Figure 19. After opening the targeted file in the left window, one simply selects the files to merge and click on Merge, at the top of the screen. This will create a new file containing all the data from the previously selected files.

\section{E-Recovery}

If an experiment is aborted, not all data will be lost, but only a txt file will be created. While useful, it cannot be opened by E-DataAid, but E-Recovery can transform it into an .edat file. Its use is quite simple: select the .txt file, click on Recover and a new file will be created.

\section{Conclusion}

E-Prime is an intuitive and easy program to use after only a short period of time. Still, some experiments may be a little trickier to create and the following sources of information could be helpful. 
- $\quad$ E-Basic Help: to help with the E-Basic code in the In Line tool.

- $\quad$ PSTNet

web

site: http://www.pstnet.com/products/E-Prime/. Offers a section with many typical programs.

- $\quad$ http://step.psy.cmu.edu/scripts/categories.html. Offers many classic examples of experiments such as the Stroop effect or the McCollough effect.

\section{Reference}

E-Prime (2004). PSTNet [Computer software].

Manuscript received November 21, 2005

Manuscript accepted October 8, 2009 